\title{
Improving seed germination and seedling growth of guava under heat and osmotic stresses by chemical and hormonal seed treatments
}

\author{
Marjan Sadat Hosseini ${ }^{\text {(D) }}$, Seyed Morteza Zahedi2,* (D), Narjes Fahadi Hoveizeh³ (iD, Li Li (D), \\ Maryam Rafiee $^{5}$ (D), Muhammad Farooq 6 (D) \\ 1. Agricultural Biotechnology Research Institute of Iran - Isfahan Branch - Agricultural Research, Education and Extension \\ Organization - Tehran, Iran \\ 2. University of Maragheh - Faculty of Agriculture - Department of Horticultural Science - Maragheh, Iran. \\ 3. Shahid Chamran University - College of Agriculture - Department of Horticultural Science - Ahvaz, Iran. \\ 4. Zhejiang University - College of Biosystems Engineering and Food Science - Xihu District Hangzhou, China. \\ 5. Urmia University - Faculty of Agricultural Sciences - Department of Horticultural Sciences - Urmia, Iran. \\ 6. Sultan Qaboos University - College of Agricultural and Marine Sciences - Department of Plant Sciences - Al-Khoud Oman.
}

\begin{abstract}
This study, consisting of two independent experiments, was conducted to optimize presowing guava seed treatments and evaluate the optimized treatments in improving germination and seedling growth of guava under heat and osmotic stresses. In the first experiment, seeds of guava cultivar White Flesh Local I were soaked in water, gibberellic acid $\left(\mathrm{GA}_{3}\right)(0.05$ and $0.1 \%)$, hydrochloric acid $(\mathrm{HCl})(5$ and $10 \%)$ and sulfuric acid $\left(\mathrm{H}_{2} \mathrm{SO}_{4}\right)(5$ and $10 \%)$ for 24 and $48 \mathrm{~h}$ (for water and $\mathrm{GA}_{3}$ ), and 2 and $5 \mathrm{~min}$ (for $\mathrm{HCl}_{\text {and }} \mathrm{H}_{2} \mathrm{SO}_{4}$ ). Seed soaking (priming) with $\mathrm{GA}_{3}(0.1 \%$, at $48 \mathrm{~h})$ and $\mathrm{HCl}(10 \%, 2 \mathrm{~min})$ were the most effective treatments to improve seed germination and early seed growth of guava and were used in the second experiment. In the second experiment, treated and untreated seeds were sown in plastic boxes between two layers of filter papers maintained at osmotic potentials of $0,-1.5$ and $-3 \mathrm{MPa}$. The germination boxes were incubated at optimal $\left(25^{\circ} \mathrm{C}\right)$ and higher $\left(32^{\circ} \mathrm{C}\right)$ temperatures. Germination was significantly suppressed at a higher temperature and with an increase in the osmotic potential. However, seed treatments with $\mathrm{GA}_{3}$ and $\mathrm{HCl}$ were effective to improve the germination and seedling growth of guava under both temperature and osmotic stresses. In conclusion, chemical and hormonal seed treatments may help improve the seed germination and seedling growth of guava under heat and osmotic stresses by modulation of antioxidant enzymes and leaf proline. Seed treatment with $\mathrm{GA}_{3}(0.1 \%, 48 \mathrm{~h})$ was the most effective in this regard.
\end{abstract}

Key word: osmotic stress, heat, germination, enzymatic antioxidants, seed priming.
Received:

Apr. 11, 2020

Accepted:

Jul. 21, 2020

Section Editor:

Hector Valenzuela

${ }^{\star}$ Corresponding author: s.m.zahedi@maragheh.ac.ir

\section{INTRODUCTION}

Guava (Psidium guajava L.), from the Myrtaceae family, is a fruit crop in tropical and subtropical regions, of economic and nutritional importance, and its fruit is used as fresh food and in processing industries to prepare fruit juices, compotes, essential oils, and powders. In addition to its specific taste and aroma guava fruits are rich in vitamin C, pectin, and minerals. Owing to its unique nutritional value, it is often referred to as "the apple of the tropics" (Dhara et al. 2017). In Iran, guava is cultivated in many tropical provinces such as Hormozgan and Sistan and Baluchestan (Khosravi et al. 2018).

Although it is propagated through cuttings, stooling, grafting, or air layering, propagation through seeds is the most common method in Iran (and in some other regions) (Shekafandeh and Khoushkhoui 2005). Propagation through seeds is also required in breeding and genetic improvement programs and to produce rootstocks (Kalyani et al. 2014). 
However, propagation through seeds may have some limitations including seed dormancy and abiotic stresses (e.g., salinity, drought, and heat stresses) in some crop plants (Zhang et al. 2015; Muhie et al. 2018).

Seed dormancy is a prerequisite for the preservation and cultivation of crops whereas genetic and environmental conditions affect the seed vigor. Therefore, seed dormancy and vigor can affect growth and development. Some physiological and biochemical stimuli affect the seed dormancy period. However, seed priming may help breaking the seed dormancy. Therefore, various seed priming treatments can be potentially used to increase the uniformity of germination (Rao et al. 2019). Seed dormancy in guava may result in poor and erratic germination (Doijode 2001; Sourabh et al. 2018). Seed dormancy may be caused by a hard seed coat making it impermeable to water and gases, and poses physical hindrance in germination.

Different methods such as water soaking, scarification, and chemical treatment are used to break seed dormancy and to stimulate germination and seedling growth (Brijwal and Kumar 2013; Dawood 2018). Acid scarification of seeds helps to break seed dormancy and improves germination (Essien 2004; Maldonado-Arciniegas et al. 2018). Scarification with mineral acids can effectively make the seed coat permeable to water and oxygen. For instance, Essien (2004) noted that seed scarification with sulphuric, nitric, and hydrochloric acid were effective to break seed dormancy of guava. However, the use of plant growth regulators, like gibberellins, may also release seed dormancy (Kalyani et al. 2014).

Germination is strongly affected by environmental conditions including temperature, water availability, light, etc. (He et al. 2013; Humphries et al. 2018). Imbibition of water is the first step in the germination process. Any deficiency of water during germination strongly limits seed germination (Cavallaro et al. 2014; Lamichhane et al. 2018). Since the 1970s, there have been many intense or long-term droughts in the arid and semiarid regions (Dai 2011). In these areas, the soil seldom maintains its field water capacity, and plants are often exposed to high temperatures and drought stresses (Jaleel et al. 2009; Gholami and Zahedi 2019).

Certain osmolytes may be used to simulate drought using solutions with different osmotic potentials (Ibrahim et al. 2001). The use of polyethylene glycol (PEG) is the most common practice used to induce osmotic stress during germination (Ibrahim et al. 2001; Sheteiwy et al. 2018).

Evaluating the effect of abiotic stresses on seed germination and seedling growth can help to elucidate the mechanisms of stress tolerance at the early stages of plant growth (Ma et al. 2016). Under stressful conditions, plants produce soluble substances with a low molecular weight named compatible solutes. These substances, including amino acids (proline and glycine), sugars (sucrose and glucose), sugar alcohols (mannitol and sorbitol), ions, organic acids, amide, amines and betaine, do not interfere with the normal plant metabolism (Slama et al. 2015). Accumulation of these solutes may help plants to survive under drought stress (Gholami and Zahedi 2019).

Germination rate usually increases linearly with temperature up to an optimal range and declines rapidly after that (Fallahi et al. 2015). A few studies on guava indicated that a temperature range of $20-30^{\circ} \mathrm{C}$ or that a constant temperature of $25^{\circ} \mathrm{C}$ is optimum for guava seed germination (Santos et al. 2015). Guava seeds exhibit erratic germination with little or no germination under normal conditions due to seed dormancy. The exact type of dormancy responsible for this has not been investigated. However, chemical pretreatments could make hard seeds capable to imbibe water and germinate especially under stress conditions (Essien 2004; Sourabh et al. 2018). Although, chemical treatments have been found effective in breaking seed dormancy and improving early seedling growth of several plant species (Grzesik et al. 2017; Kim 2019), the influence of these treatments has not been evaluated under less than optimal conditions. This study was, therefore, conducted to evaluate the potential of chemical and hormonal seed treatments in improving seed germination and seedling growth of guava under heat and osmotic stresses.

\section{MATERIAL AND METHODS}

\section{Plant material and experimental detail}

Seeds of guava variety "White flesh local I" were collected from a commercial guava orchard in Bandar Abbas, Iran. This variety is endemic and popular in the Hormozgan province $\left(56^{\circ} 26^{\prime} \mathrm{E}, 27^{\circ} 17^{\prime} \mathrm{N}\right)$ of Iran. Fresh guava seed cores were 
obtained from local fresh-cut fruit. Seed cores were placed in a blender, excess water was added; and then seeds were separated from the fruit, cleaned, and dried in shadow (one week).

All seeds were pretreated with a $0.1 \%$ sodium hypochlorite $(\mathrm{NaOCl})$ solution for $5 \mathrm{~min}$ and then subsequently rinsed with distilled water and air dried to avoid fungal attack. The study consisted of two independent experiments.

\section{Experiment I}

Guava seeds were soaked in distilled water, $\mathrm{GA}_{3}(0.05$ and $0.1 \%), \mathrm{HCl}(5$ and $10 \%)$, and $\mathrm{H}_{2} \mathrm{SO}_{4}$ (5 and 10\%). Seeds were soaked in water and $\mathrm{GA}_{3}$ for 24 and $48 \mathrm{~h}$, and were soaked in $\mathrm{HCl}$ and $\mathrm{H}_{2} \mathrm{SO}_{4}$ for 2 and 5 min maintaining a seed to solution ratio of 1:5 (w/v). After treatment, seeds were rinsed with distilled water and air dried at $20^{\circ} \mathrm{C}$ under shade until reaching original weight. Seeds (30) were placed evenly on sterilized filter paper moistened with $15 \mathrm{~mL}$ of distilled water in Petri dishes $(12 \mathrm{~cm}$ ). The filter papers were replaced every three days to ensure even moisture. Dishes were sealed with Parafilm to minimize water loss from evaporation. Petri dishes were closed to prevent moisture loss and avoid contamination and were placed in a germinator at $25^{\circ} \mathrm{C}$. The experiment was conducted as a factorial completely randomized design with three replications.

\section{Experiment II}

Based on the results of experiment I, guava seeds were soaked in distilled water, $10 \% \mathrm{HCl}$ for $2 \mathrm{~min}$, and $\mathrm{GA}_{3}(0.1 \%$, $48 \mathrm{~h}$ ) maintaining a seed to solution ratio of 1:5. The treated seeds were rinsed with distilled water and air dried at $20^{\circ} \mathrm{C}$ under shade until the original weight $(\sim 14 \%)$. Seeds (30 in each box) were germinated in plastic boxes between two layers of filter papers moistened with $15 \mathrm{~mL}$ of distilled water $(0 \mathrm{MPa})$ and with solutions of polyethylene glycol $\left(\mathrm{PEG}_{6000}\right)$ having an osmotic potential -1.5 and $-3.0 \mathrm{MPa}$. The target osmotic potential solutions were prepared following Michel and Kaufmann (1973). Distilled water, containing 0.02\% fungicide (Captan; $\mathrm{C}_{9} \mathrm{H}_{8} \mathrm{C}_{13} \mathrm{NO}_{2} \mathrm{~S}$ ) was used to prepare the solutions. The boxes were placed in a germinator at 25 (optimal) and $32{ }^{\circ} \mathrm{C}$ (heat stress) with $10 / 14 \mathrm{~h}$ day/night duration. The filter papers, in each box, were replaced every two days to maintain a uniform water potential. The experiment was conducted as a factorial completely randomized design with three replications.

\section{Observations and measurements}

\section{Germination and seedling growth}

The number of germinated seeds was counted daily until reaching a constant count. Both experiments were terminated 30 days after sowing. Seeds with a radicle length of $2 \mathrm{~mm}$ were considered as germinated. Germination percentage was calculated as the ratio of the number of germinated seeds to the number of seeds planted and was expressed as a percentage. Germination rate was estimated using the following equation by Ranal et al. (2009) (Eq. 1).

$$
\mathrm{R}_{\mathrm{s}}=\sum_{i=1}^{n} \frac{S i}{D i}
$$

where $R_{s}$ is the germination rate, $S_{i}$ is the number of germinated seeds per day, $D_{i}$ is the number of days passed since the beginning of the experiment.

At the final harvest, five seedlings were randomly chosen from each replication to record morphological parameters. Root and shoot length were determined. Root and shoots were separated, and fresh weight was recorded immediately and was then oven-dried at $70{ }^{\circ} \mathrm{C}$ for $24 \mathrm{~h}$ to record dry weight. A digital weighing balance, with a precision of $0.001 \mathrm{~g}$, was used to record fresh and dry weights. Leaf area was recorded using the image software Image J (National Institutes of Health, Maryland, USA). 


\section{Proline extraction and measurement}

Total free proline contents were measured following a modified method of Khare et al. (2012). Samples ( $0.1 \mathrm{~g}$ of root and shoot) were homogenized in $0.5 \mathrm{~mL}$ of $3 \%$ (w/v) sulphosalicylic acid. Each homogenate $(0.2 \mathrm{~mL})$ was combined with $0.2 \mathrm{~mL}$ of glacial acetic acid to which, $0.2 \mathrm{~mL}$ of ninhydrin was added. The mixture was boiled in a water bath at $100{ }^{\circ} \mathrm{C}$ for $30 \mathrm{~min}$ and cooled in an ice bath immediately. The chromophore containing toluene was separated and absorbance of the red color was read at $520 \mathrm{~nm}$ on the UV-visible spectrophotometer (Cary $100 \mathrm{UV}$-Visible Spectrophotometer, Agilent, United States).

\section{Total soluble carbohydrate}

The soluble carbohydrate contents were estimated using the Anthrone method (Yemm and Willis 1954). The sample ( $0.5 \mathrm{~g}$ each of root and shoot) was homogenized with hot aqueous ethanol (80\%). After centrifugation, $0.2 \mathrm{~mL}$ of supernatant was transmitted into another test tube and distilled water was added to reach a volume of $1 \mathrm{~mL}$. Anthrone reagent $(0.2 \%)$ was then added into the tubes. The samples were heated in a boiling water bath for $8 \mathrm{~min}$ and were then cooled rapidly; the intensity of the green to dark green color was estimated at $625 \mathrm{~nm}$ by a digital spectrophotometer (Cary $100 \mathrm{UV}$-Visible Spectrophotometer, Agilent, United States).

\section{Enzymatic antioxidants and oxidative stress markers}

Guava seedling samples were ground in liquid nitrogen and the powder was extracted with $10 \mathrm{~mL}$ of $50 \mathrm{mmol} \cdot \mathrm{L}^{-1}$ phosphate buffer ( $\mathrm{pH} 7.0$ ), then centrifuged at $14,000 \times \mathrm{g}$ for $15 \mathrm{~min}$ at $4{ }^{\circ} \mathrm{C}$. Peroxidase (POD) activity was assayed spectrophotometrically at $470 \mathrm{~nm}$ using guaiacol as a substrate according to Hemeda and Klein (1990). Superoxide dismutase (SOD) activity was measured by estimating its ability to prevent the photochemical reduction of nitro blue tetrazolium (NBT) according to Beauchamp and Fridovich (1971). The SOD activity was measured spectrophotometrically at $560 \mathrm{~nm}$. The catalase (CAT) activity was measured following the method of Aebi (1974). Tissues $(0.1 \mathrm{~g})$ were disrupted in $0.4 \mathrm{~mL}$ of extracting buffer $\left(50 \mathrm{mmol} \cdot \mathrm{L}^{-1}\right.$ phosphate buffer, $\mathrm{pH}$ 7, and $1 \%$ Triton X-100). The homogenates were centrifuged for $20 \mathrm{~min}$ at $12,000 \times \mathrm{g}$. Then, $0.1 \mathrm{~mL}$ of the supernatant was mixed with $0.9 \mathrm{~mL}$ of the buffered substrate $\left(20 \mathrm{mmol} \cdot \mathrm{L}^{-1} \mathrm{H}_{2} \mathrm{O}_{2}\right.$ in $50 \mathrm{mmol} \cdot \mathrm{L}^{-1}$ phosphate buffer, $\mathrm{pH}$ 7), and the OD of the mixture was measured spectrophotometrically at $240 \mathrm{~nm}$.

Hydrogen peroxide $\left(\mathrm{H}_{2} \mathrm{O}_{2}\right)$ was determined spectrophotometrically at $390 \mathrm{~nm}$ according to Alexieva et al. (2001) to quantify $\mathrm{H}_{2} \mathrm{O}_{2}$ content. The accumulation of malondialdehyde (MDA) in leaf samples due to lipid peroxidation was measured spectrophotometrically at $532 \mathrm{~nm}$ following Dhindsa et al. (1981).

\section{Statistical analyses}

The data were statistically analyzed by analysis of variance using the Statistical Analysis Software (SAS, version 9.1). Duncan's new multiple range test was used for mean separation at a significance level of $\mathrm{p}<0.05$. Principal component analysis, Pearson correlation coefficient, and dendrogram clustering were carried out using statistical software R v3.4.3.

\section{RESULTS}

\section{Experiment I}

All seed priming and soaking treatments significantly improved the germination and germination rate of guava seeds as compared to the control (Table 1). In this regard, the highest germination percentage (93.97\%) and germination rate (21.1) were observed with seed priming by $\mathrm{GA}_{3}(0.1 \%$ for $48 \mathrm{~h}$ ), followed by seed soaking in $\mathrm{HCl}$ ( $10 \%$ for $2 \mathrm{~min}$ ) (Table 1 ). 
Table 1. Influence of chemical and hormonal seeds treatments on germination and seedling growth of guava.

\begin{tabular}{|c|c|c|c|c|c|c|c|c|c|}
\hline Treatments & Time & $\begin{array}{l}\text { GM } \\
(\%)\end{array}$ & GMR & $\begin{array}{c}\mathrm{RL} \\
(\mathrm{cm})\end{array}$ & $\begin{array}{c}\mathrm{PL} \\
(\mathrm{cm})\end{array}$ & $\begin{array}{l}\text { PLTL } \\
(\mathrm{cm})\end{array}$ & $\begin{array}{c}\text { RDW } \\
\text { (g) }\end{array}$ & $\begin{array}{c}\text { SDW } \\
\text { (g) }\end{array}$ & $\begin{array}{l}\text { PLTTW } \\
\text { (g) }\end{array}$ \\
\hline \multirow{2}{*}{ Water } & $24 \mathrm{~h}$ & $53.70 \mathrm{i}$ & $10.53 n$ & $1.00 \mathrm{j}$ & $1.79 \mathrm{i}$ & $2.79 \mathrm{~m}$ & $4.60 \mathrm{j}$ & 9.101 & $13.76 \mathrm{~m}$ \\
\hline & $48 \mathrm{~h}$ & $64.90 \mathrm{~h}$ & $11.26 \mathrm{~m}$ & $1.02 \mathrm{j}$ & $1.99 \mathrm{~h}$ & 3.011 & $4.70 \mathrm{j}$ & $9.30 \mathrm{k}$ & 14.031 \\
\hline \multirow{2}{*}{$\mathrm{GA}_{3}(0.05 \%)$} & $24 \mathrm{~h}$ & $66.23 \mathrm{~h}$ & $12.63 \mathrm{k}$ & $1.25 \mathrm{i}$ & $2.01 \mathrm{~h}$ & $3.22 \mathrm{k}$ & $5.21 \mathrm{i}$ & $10.32 \mathrm{~h}$ & $15.40 \mathrm{j}$ \\
\hline & $48 \mathrm{~h}$ & $71.57 \mathrm{~g}$ & $13.40 \mathrm{j}$ & $1.37 \mathrm{~h}$ & $2.49 \mathrm{f}$ & $3.86 \mathrm{i}$ & $6.28 \mathrm{~h}$ & $10.79 \mathrm{~g}$ & $17.09 \mathrm{~h}$ \\
\hline \multirow{2}{*}{$\mathrm{GA}_{3}(0.1 \%)$} & $24 \mathrm{~h}$ & $84.47 b c$ & $17.79 \mathrm{e}$ & $2.13 d$ & $3.06 \mathrm{~d}$ & $5.2 \mathrm{f}$ & 8.19 e & $11.78 \mathrm{f}$ & $19.97 \mathrm{e}$ \\
\hline & $48 \mathrm{~h}$ & $93.97 a$ & $21.10 \mathrm{a}$ & $2.49 a$ & $4.79 a$ & $7.29 a$ & $13.73 a$ & $17.23 a$ & $31.00 \mathrm{a}$ \\
\hline \multirow{2}{*}{$\mathrm{HCl}(5 \%)$} & $2 \min$ & 76.40 ef & $15.8 \mathrm{~h}$ & $1.58 \mathrm{~g}$ & $2.48 \mathrm{f}$ & $4.07 \mathrm{~h}$ & $7.13 \mathrm{~g}$ & $10.77 \mathrm{~g}$ & $17.88 \mathrm{~g}$ \\
\hline & $5 \mathrm{~min}$ & $78.72 \mathrm{de}$ & $16.36 \mathrm{~g}$ & $1.80 \mathrm{f}$ & $2.81 \mathrm{e}$ & $4.62 \mathrm{~g}$ & $7.83 \mathrm{f}$ & $10.70 \mathrm{~g}$ & $18.52 \mathrm{f}$ \\
\hline \multirow{2}{*}{$\mathrm{HCl}(10 \%)$} & $2 \min$ & $91.67 a$ & $19.79 \mathrm{~b}$ & $2.36 \mathrm{~b}$ & $4.72 a$ & $7.06 \mathrm{~b}$ & $12.21 \mathrm{~b}$ & $15.79 \mathrm{~b}$ & $27.97 \mathrm{~b}$ \\
\hline & $5 \mathrm{~min}$ & $87.63 \mathrm{~b}$ & $19.22 \mathrm{c}$ & $2.31 \mathrm{~b}$ & $3.79 \mathrm{~b}$ & $6.09 c$ & $9.93 \mathrm{c}$ & $13.12 \mathrm{c}$ & $23.17 c$ \\
\hline \multirow{2}{*}{$\mathrm{H}_{2} \mathrm{SO}_{4}(5 \%)$} & $2 \min$ & $81.52 \mathrm{~cd}$ & $16.77 \mathrm{f}$ & $2.05 \mathrm{e}$ & $3.52 \mathrm{c}$ & $5.53 d$ & $8.34 \mathrm{e}$ & $11.50 \mathrm{f}$ & $19.80 \mathrm{e}$ \\
\hline & $5 \min$ & $85.78 \mathrm{~b}$ & $18.50 \mathrm{~d}$ & $2.25 \mathrm{c}$ & $3.03 \mathrm{~d}$ & $5.27 \mathrm{e}$ & $8.73 \mathrm{~d}$ & $12.19 \mathrm{~d}$ & $20.91 \mathrm{~d}$ \\
\hline \multirow{2}{*}{$\mathrm{H}_{2} \mathrm{SO}_{4}(10 \%)$} & $2 \min$ & $74.73 \mathrm{gf}$ & $14.64 i$ & $1.37 \mathrm{~h}$ & $2.70 \mathrm{e}$ & $4.08 \mathrm{~h}$ & $6.13 \mathrm{~h}$ & $10.16 \mathrm{i}$ & $16.22 \mathrm{i}$ \\
\hline & $5 \mathrm{~min}$ & $72.59 \mathrm{~g}$ & $12.29 \mathrm{I}$ & $1.23 \mathrm{i}$ & $2.19 \mathrm{~g}$ & $3.43 \mathrm{j}$ & $4.62 \mathrm{j}$ & $9.79 \mathrm{j}$ & $14.49 \mathrm{k}$ \\
\hline
\end{tabular}

Means sharing the same letters, for a parameter, don't differ significantly different at $p<0.05$ as per Duncan's new multiple range test. GM: germination; GMR: germination rate; RL: radicle length, PL: plumule length; PLTL: plantlet length; RDW: root dry weight; SDW: shoot dry weight; PLTTW: Total plantlet weight; $\mathrm{GA}_{3}$ : gibberellic acid.

All seed priming and soaking treatments significantly improved radicle and plumule length and dry weight, and total plantlet length and weight of guava than water soaking alone (Table 1). The highest radicle length $(2.49 \mathrm{~cm})$ and plumule length $(4.79 \mathrm{~cm})$ was observed in $\mathrm{GA}_{3}$ seed priming by $0.1 \%$ for $48 \mathrm{~h}$. Likewise, the highest plantlet length $(7.29 \mathrm{~cm})$ and the total weight of plantlet $(31 \mathrm{~g})$ were recorded with $\mathrm{GA}_{3}$ seed priming $(0.1 \%$ for $48 \mathrm{~h})$ (Table 1$)$. Likewise, the highest root and shoot dry weights were also recorded with $\mathrm{GA}_{3}$ seed priming $(0.1 \%$ for $48 \mathrm{~h})$.

\section{Experiment II}

\section{Germination percentage and rate}

Osmotic stress significantly reduced the germination percentage and germination rate of guava seeds with an increase in the level of stress (Table 2). Heat stress $\left(32^{\circ} \mathrm{C}\right.$ ) was effective to promote germination percentage. However, seed priming with $\mathrm{GA}_{3}$ and soaking in $\mathrm{HCl}$ significantly improved the germination percentage and rate at all levels of osmotic stress and at both temperature regimes. In this regard, $\mathrm{GA}_{3}$ seed priming with $0.1 \%$ for $48 \mathrm{~h}$ improved the germination percentage and rate at all stress levels and at both temperature regimes (Table 2).

\section{Seedling growth parameters}

Osmotic and temperature stresses significantly reduced seedling growth parameters, namely radicle, and plumule length, root and shoot dry weight, root and shoot fresh weight and leaf area as compared to the respective controls. Seed treatments significantly improved seedling growth parameters under both stress treatments, however, $\mathrm{GA}_{3}$ seed priming was the most effective (Table 2).

\section{Enzymatic antioxidants and oxidative markers}

Activities of enzymatic antioxidants (peroxidase, superoxide dismutase and catalase) and oxidative markers (hydrogen peroxide, and malondialdehyde) significantly increased under both osmotic and temperature stresses on guava seedlings. However, seed 
Table 2. Influence of chemical and hormonal seeds treatments on germination and seedling growth of guava under different osmotic and temperature regimes.

\begin{tabular}{|c|c|c|c|c|c|c|c|c|c|c|c|}
\hline Treatments & $\begin{array}{c}\psi_{\mathrm{s}} \\
(-\mathrm{MPa})\end{array}$ & $\begin{array}{c}\text { Temp. } \\
\left({ }^{\circ} \mathrm{C}\right)\end{array}$ & $\begin{array}{l}\text { GM } \\
\text { (\%) }\end{array}$ & GMR & $\begin{array}{l}\mathrm{RL} \\
(\mathrm{cm})\end{array}$ & $\begin{array}{l}\mathrm{PL} \\
(\mathrm{cm})\end{array}$ & $\begin{array}{c}\text { RDW } \\
\text { (g) }\end{array}$ & $\begin{array}{c}\text { SDW } \\
(g)\end{array}$ & $\begin{array}{c}\text { RFW } \\
\text { (g) }\end{array}$ & $\begin{array}{c}\text { SFW } \\
\text { (g) }\end{array}$ & $\begin{array}{c}\text { LA } \\
\left(\mathrm{cm}^{2} / \text { plant }\right)\end{array}$ \\
\hline \multirow{6}{*}{ Water } & 0 & 25 & $70.77 d$ & $18.7 \mathrm{c}$ & $2.28 a b$ & $4.12 \mathrm{c}$ & $10.78 \mathrm{e}$ & $13.20 \mathrm{~d}$ & $0.214 \mathrm{e}$ & $0.299 a-c$ & $11.07 \mathrm{~d}$ \\
\hline & 0 & 32 & $68.30 \mathrm{e}$ & $18.25 \mathrm{~d}$ & $2.25 \mathrm{ab}$ & $4.02 \mathrm{~d}$ & $10.47 \mathrm{f}$ & $13.17 \mathrm{~d}$ & $0.209 \mathrm{f}$ & $0.280 a-d$ & $10.97 \mathrm{e}$ \\
\hline & 1.5 & 25 & $25.20 \mathrm{~m}$ & $5.81 I$ & $1.31 \mathrm{gh}$ & $2.03 \mathrm{~m}$ & $5.78 \mathrm{~m}$ & $9.96 n$ & $0.109 \mathrm{~m}$ & $0.106 \mathrm{f}$ & $3.89 n$ \\
\hline & 1.5 & 32 & $23.50 \mathrm{mn}$ & $5.27 \mathrm{~m}$ & $1.24 \mathrm{ghi}$ & $1.91 \mathrm{n}$ & $5.29 n$ & $9.88 n$ & $0.108 \mathrm{~m}$ & $0.082 f$ & 3.730 \\
\hline & 3.0 & 25 & $19.82 n$ & $3.72 n$ & $1.13 \mathrm{hi}$ & 1.720 & $5.00 \circ$ & 8.490 & $0.091 \mathrm{n}$ & $0.077 \mathrm{f}$ & $2.51 p$ \\
\hline & 3.0 & 32 & $19.42 n$ & $3.50 n$ & $1 \mathrm{i}$ & $1.63 p$ & $4.38 p$ & $8.08 p$ & $0.084 p$ & $0.282 a-d$ & $1.72 \mathrm{q}$ \\
\hline \multirow{6}{*}{$\mathrm{HCl}$} & 0 & 25 & $87.17 \mathrm{~b}$ & $19.82 b$ & $2.35 a$ & $4.71 \mathrm{a}$ & $12.34 \mathrm{c}$ & $15.82 \mathrm{c}$ & $0.262 c$ & $0.325 a b$ & $11.66 \mathrm{~b}$ \\
\hline & 0 & 32 & $76.23 \mathrm{~b}$ & $19.58 \mathrm{~b}$ & $2.34 a$ & $4.43 b$ & $11.23 d$ & $15.20 \mathrm{~cd}$ & $0.224 d$ & $0.312 a-c$ & $11.24 \mathrm{c}$ \\
\hline & 1.5 & 25 & $51.43 \mathrm{~h}$ & $11.59 \mathrm{~g}$ & $1.97 \mathrm{~cd}$ & $3.54 \mathrm{~g}$ & $9.56 \mathrm{~g}$ & $11.60 \mathrm{i}$ & $0.195 \mathrm{~g}$ & $0.177 b-f$ & $8.67 \mathrm{~h}$ \\
\hline & 1.5 & 32 & $48.50 \mathrm{i}$ & $11.26 \mathrm{~g}$ & $1.94 \mathrm{~cd}$ & $3.43 \mathrm{~h}$ & $9.26 \mathrm{~h}$ & $11.20 \mathrm{j}$ & $0.187 \mathrm{~h}$ & $0.161 b-f$ & $7.56 \mathrm{i}$ \\
\hline & 3.0 & 25 & $32.43 \mathrm{k}$ & $7.18 \mathrm{j}$ & $1.63 \mathrm{ef}$ & $2.35 \mathrm{k}$ & $7.63 \mathrm{k}$ & $10.34 I$ & $0.132 \mathrm{k}$ & $0.126 \mathrm{~d}-\mathrm{f}$ & 4.251 \\
\hline & 3.0 & 32 & 27.501 & $6.27 \mathrm{k}$ & $1.47 \mathrm{fg}$ & $2.14 I$ & 6.481 & $10.20 \mathrm{~m}$ & 0.127 I & 0.112 ef & $4.00 \mathrm{~m}$ \\
\hline \multirow{6}{*}{$\mathrm{GA}_{3}$} & 0 & 25 & $91.63 a$ & $20.77 a$ & $2.50 \mathrm{a}$ & $4.76 a$ & $13.77 \mathrm{a}$ & $17.14 \mathrm{a}$ & $0.274 a$ & $0.434 a$ & $11.97 a$ \\
\hline & 0 & 32 & $87.87 b$ & $20.71 a$ & $2.43 a$ & $4.75 \mathrm{a}$ & $13.27 b$ & $17.00 \mathrm{~b}$ & $0.267 b$ & $0.427 a$ & $11.93 a$ \\
\hline & 1.5 & 25 & $59.10 \mathrm{f}$ & $17.5 \mathrm{e}$ & $1.79 \mathrm{cde}$ & $3.84 \mathrm{e}$ & $10.38 \mathrm{f}$ & $12.60 \mathrm{e}$ & $0.200 \mathrm{~g}$ & $0.274 \mathrm{a}-\mathrm{e}$ & $9.89 \mathrm{f}$ \\
\hline & 1.5 & 32 & $56.67 \mathrm{~g}$ & $15.3 \mathrm{f}$ & $2.04 b c$ & $3.68 \mathrm{f}$ & $9.69 \mathrm{~g}$ & $11.84 \mathrm{~h}$ & $0.198 \mathrm{~g}$ & $0.189 b-f$ & $9.81 \mathrm{~g}$ \\
\hline & 3.0 & 25 & $38.30 \mathrm{j}$ & $8.44 \mathrm{~h}$ & $1.81 \mathrm{cde}$ & $2.92 \mathrm{i}$ & $8.52 \mathrm{i}$ & $10.90 \mathrm{k}$ & $0.167 \mathrm{i}$ & $0.155 c-f$ & $5.49 \mathrm{j}$ \\
\hline & 3.0 & 32 & $37.20 \mathrm{jk}$ & $7.82 \mathrm{i}$ & $1.75 \mathrm{de}$ & $2.74 \mathrm{j}$ & $8.32 \mathrm{j}$ & 10.531 & $0.156 j$ & $0.146 c-f$ & $4.89 \mathrm{k}$ \\
\hline
\end{tabular}

Means sharing the same letters, for a parameter, don't differ significantly different at $\mathrm{p}<0.05$ as per Duncan's new multiple range test. GM: germination; GMR: germination rate; RL: radicle length, PL: plumule length; RDW: root dry weight; SDW: shoot dry weight; RFW: root fresh weight; SFW: shoot fresh weight; $\mathrm{LA}$ : leaf area; $\mathrm{HCl}$ : seed soaking in $10 \% \mathrm{HCl}$ solution for $2 \mathrm{~min} ; \mathrm{GA}_{3}$ : seed priming in $0.1 \%$ gibberellic acid solution for $48 \mathrm{~h}$.

treatments further increased the activity of enzymatic antioxidants under both stresses. Nonetheless, seed treatments caused a significant reduction in hydrogen peroxide and malondialdehyde. Seed priming with $\mathrm{GA}_{3}$ was the most effective treatment in increasing the activity of enzymatic antioxidants while decreasing for hydrogen peroxide and malondialdehyde (Table 3).

\section{Proline and carbohydrate contents}

Proline and carbohydrate contents in guava seedlings (shoot and root) significantly increased under both osmotic and temperature stresses. Seed treatments further improved the proline and carbohydrate contents under both stresses, however, seed priming with $\mathrm{GA}_{3}$ was the most effective (Fig. 1).

\section{Principal component analysis, correlation and dendrogram clustering of guava seeds under osmotic conditions}

All morphological and biochemical traits were loaded into two principal components (PC1 and PC2), explaining 94.50 of the total variances (Fig. 2a). Most of the examined traits were discriminated by $\mathrm{PC1}$, and thus explained by the larger proportions of variances (78.30\%); while the lower proportions of variances (16.2\%) were indicated by PC2 in experiment II (Fig. 2a). Principal component analysis (PCA) plots separated the treatment groups into three main groups: (i) guava seeds under -1.5 and $-3 \mathrm{MPa}$ (osmotic stress) at 25 and $32^{\circ} \mathrm{C}$, (ii) guava seeds treated with $\mathrm{HCl}$ soaking $(10 \%, 2 \mathrm{~min})$ and $\mathrm{GA}_{3}$ priming $(0.1 \%, 48 \mathrm{~h})$ under -1.5 and $-3 \mathrm{MPa}$ at 25 and $32^{\circ} \mathrm{C}$, (iii) guava seeds soaked in water, $\mathrm{HCl}(10 \%, 2 \mathrm{~min})$ and $\mathrm{GA}_{3}(0.1 \%, 48 \mathrm{~h})$ without osmotic stress at 25 and $32^{\circ} \mathrm{C}$ (Fig. $2 \mathrm{~b}$ ). All the enzyme activities were positively associated with $\mathrm{GA}_{3}$ priming $\left(0.1 \%, 48 \mathrm{~h}\right.$ at 25 and $\left.32^{\circ} \mathrm{C}\right)$ under osmotic stress, while $\mathrm{H}_{2} \mathrm{O}_{2}$ were positively linked with guava seeds under -1.5 and $-3 \mathrm{MPa}$ (osmotic stress) at 25 and $32^{\circ} \mathrm{C}$ (Fig. 2c). In addition, germination and seedling growth parameters were positively associated with $\mathrm{GA}_{3}$ priming $(0.1 \%, 48 \mathrm{~h})$ at 25 and $32{ }^{\circ} \mathrm{C}$ (Fig. $2 \mathrm{c}$ ). 
Table 3. Influence of chemical and hormonal seeds treatments on activities of antioxidant enzymes, hydrogen peroxide and malondialdehyde contents in guava under different osmotic and temperature regimes.

\begin{tabular}{|c|c|c|c|c|c|c|c|}
\hline Treatments & $\begin{array}{c}\psi_{s} \\
(-\mathrm{MPa})\end{array}$ & $\begin{array}{l}\text { Temp. } \\
\left({ }^{\circ} \mathrm{C}\right)\end{array}$ & $\begin{array}{c}\text { POD } \\
\text { (U/g. FW. } \mathrm{min} \text { ) }\end{array}$ & $\begin{array}{c}\text { SOD } \\
\text { (U/g. FW. h) }\end{array}$ & $\begin{array}{c}\text { CAT } \\
\text { (U/g. FW. min) }\end{array}$ & $\begin{array}{c}\mathrm{H}_{2} \mathrm{O}_{2} \\
\left(\mathrm{nmol} \cdot \mathrm{g}^{-1} \mathrm{FW}\right)\end{array}$ & $\begin{array}{c}\text { MDA } \\
\left(\mathrm{nmol} \cdot \mathrm{g}^{-1} \mathrm{FW}\right)\end{array}$ \\
\hline \multirow{6}{*}{ Water } & 0 & 25 & 64.520 & $8.38 n$ & $6.13 p$ & $0.279 n$ & $0.021 \mathrm{i}-\mathrm{I}$ \\
\hline & 0 & 32 & $71.17 n$ & $13.58 \mathrm{~m}$ & 7.390 & $0.318 \mathrm{~m}$ & $0.024 \mathrm{ijk}$ \\
\hline & 1.5 & 25 & $115.85 \mathrm{j}$ & $24.17 i$ & $15.13 \mathrm{j}$ & $0.957 \mathrm{~d}$ & $0.071 \mathrm{~cd}$ \\
\hline & 1.5 & 32 & $123.39 \mathrm{i}$ & $25.46 \mathrm{i}$ & $15.25 \mathrm{j}$ & $1.272 \mathrm{c}$ & $0.077 \mathrm{c}$ \\
\hline & 3.0 & 25 & $150.93 \mathrm{fg}$ & $28.46 \mathrm{fg}$ & $19.84 \mathrm{~g}$ & $1.656 \mathrm{~b}$ & $0.089 \mathrm{~b}$ \\
\hline & 3.0 & 32 & $155.35 \mathrm{f}$ & 29.77 ef & $21.82 \mathrm{f}$ & $1.927 a$ & $0.103 a$ \\
\hline \multirow{6}{*}{$\mathrm{HCl}$} & 0 & 25 & $83.51 \mathrm{~m}$ & $14.34 \mathrm{Im}$ & $8.75 n$ & $0.247 p$ & $0.014 \mathrm{klm}$ \\
\hline & 0 & 32 & $90.26 I$ & $15.52 I$ & $9.12 \mathrm{~m}$ & 0.249 o & $0.018 \mathrm{j}-\mathrm{m}$ \\
\hline & 1.5 & 25 & $131.55 \mathrm{~h}$ & 26.04 hi & $16.34 \mathrm{i}$ & $0.471 \mathrm{j}$ & $0.041 \mathrm{gh}$ \\
\hline & 1.5 & 32 & $147.22 \mathrm{~g}$ & $27.58 \mathrm{gh}$ & $18.85 \mathrm{~h}$ & $0.493 i$ & $0.047 \mathrm{~g}$ \\
\hline & 3.0 & 25 & $173.66 \mathrm{e}$ & $31.16 \mathrm{de}$ & $23.13 \mathrm{e}$ & $0.655 \mathrm{f}$ & $0.063 \mathrm{de}$ \\
\hline & 3.0 & 32 & $186.99 \mathrm{~d}$ & $32.33 \mathrm{~d}$ & 23.56 & $0.861 \mathrm{e}$ & 0.068 cde \\
\hline \multirow{6}{*}{$\mathrm{GA}_{3}$} & 0 & 25 & 96.17 I & $18.42 \mathrm{k}$ & $10.65 I$ & $0.229 r$ & $0.009 \mathrm{~m}$ \\
\hline & 0 & 32 & $105.73 \mathrm{k}$ & $20.26 j$ & $12.13 \mathrm{k}$ & $0.231 \mathrm{q}$ & $0.011 \mathrm{Im}$ \\
\hline & 1.5 & 25 & $230.74 \mathrm{c}$ & $34.24 \mathrm{c}$ & $25.64 d$ & 0.387 I & $0.027 \mathrm{ij}$ \\
\hline & 1.5 & 32 & $236.62 \mathrm{c}$ & $35.78 \mathrm{bc}$ & $27.57 c$ & $0.428 \mathrm{k}$ & 0.033 hi \\
\hline & 3.0 & 25 & $251.32 \mathrm{~b}$ & $36.53 b$ & $38.71 b$ & $0.617 \mathrm{~h}$ & $0.052 \mathrm{fg}$ \\
\hline & 3.0 & 32 & $258.13 a$ & $41.19 a$ & $45.81 \mathrm{a}$ & $0.636 \mathrm{~g}$ & 0.059 ef \\
\hline
\end{tabular}

Means sharing the same letters, for a parameter, don't differ significantly different at $\mathrm{p}<0.05$ as per Duncan's new multiple range test. POD: peroxidase activity; SOD: superoxide dismutase activity; CAT: catalase; $\mathrm{H}_{2} \mathrm{O}_{2}$ : hydrogen peroxide, MDA: malondialdehyde; $\mathrm{HCl}$ : seed soaking in $10 \% \mathrm{HCl}$ solution for 2 min; $\mathrm{GA}$ : seed priming in $0.1 \%$ gibberellic acid solution for $48 \mathrm{~h}$.

\section{DISCUSSION}

Chemical (acid) and hormonal $\left(\mathrm{GA}_{3}\right)$ treatments were effective to improve the germination and early seedling growth of guava seeds. Acid scarification is an effective method for breaking seed dormancy due to hard seed coats. However, soaking seeds in concentrated acid for longer durations may damage the seed embryo. Similarly, soaking in diluted acids for short durations may not make the hard seeds permeable to oxygen and water (Tanaka-Oda et al. 2009). The same pattern was noted in this study; seed soaking in $\mathrm{HCl}(10 \%, 2 \mathrm{~min})$ was the most effective treatment to break the seed dormancy in guava. While $\mathrm{GA}_{3}$ is involved in the activation of germination process, seed soaking in $\mathrm{GA}_{3}$ was effective in improving seed germination as well.

The current research thus indicates that $\mathrm{GA}_{3}$ promotes germination in guava. In maize, a $\mathrm{GA}_{30 x}$ deletion mutant could not germinate even under normal conditions while the transcript level of $\mathrm{GA}_{30 \times 2}$ improved germination 40 -fold in dormancy-broken seeds as compared to dormant ones. Also, $\mathrm{GA}_{20 x 1}$ expression levels were higher in dormant seeds than in nondormant seeds (Li et al. 2017). Germination and seedling growth are promoted by the homeostasis of plant growth hormones (Dissanayake et al. 2010). Gibberellic acid increases the activity of the protease enzyme and changes proteins to amino acids such as tryptophan (auxin precursor). Gibberellic acid also modulates the dry weight of plants because it increases the leaf area and photosynthesis (Lester et al. 2002). Gibberellic acid functions as a regulator for plant growth through increasing cell division and cell elongation and replication (Salehi Sardoei et al. 2014). Gibberellic acid also modulates the hydrolysis of starch into monosaccharides, which promotes germination and early seedling growth (Arteca 1996; Farooq et al. 2018). It also stimulates the activation and/or de novo synthesis of hydrolytic enzymes involved in the germination metabolism (Paleg et al. 1964; Farooq et al. 2006).

Osmotic stress suppresses germination and morphological traits such as fresh and dry weight of shoots and roots and the leaf area in guava (Shaukat et al. 2015). The present study showed that severe drought stress and an increase in temperature led to the 
(a)
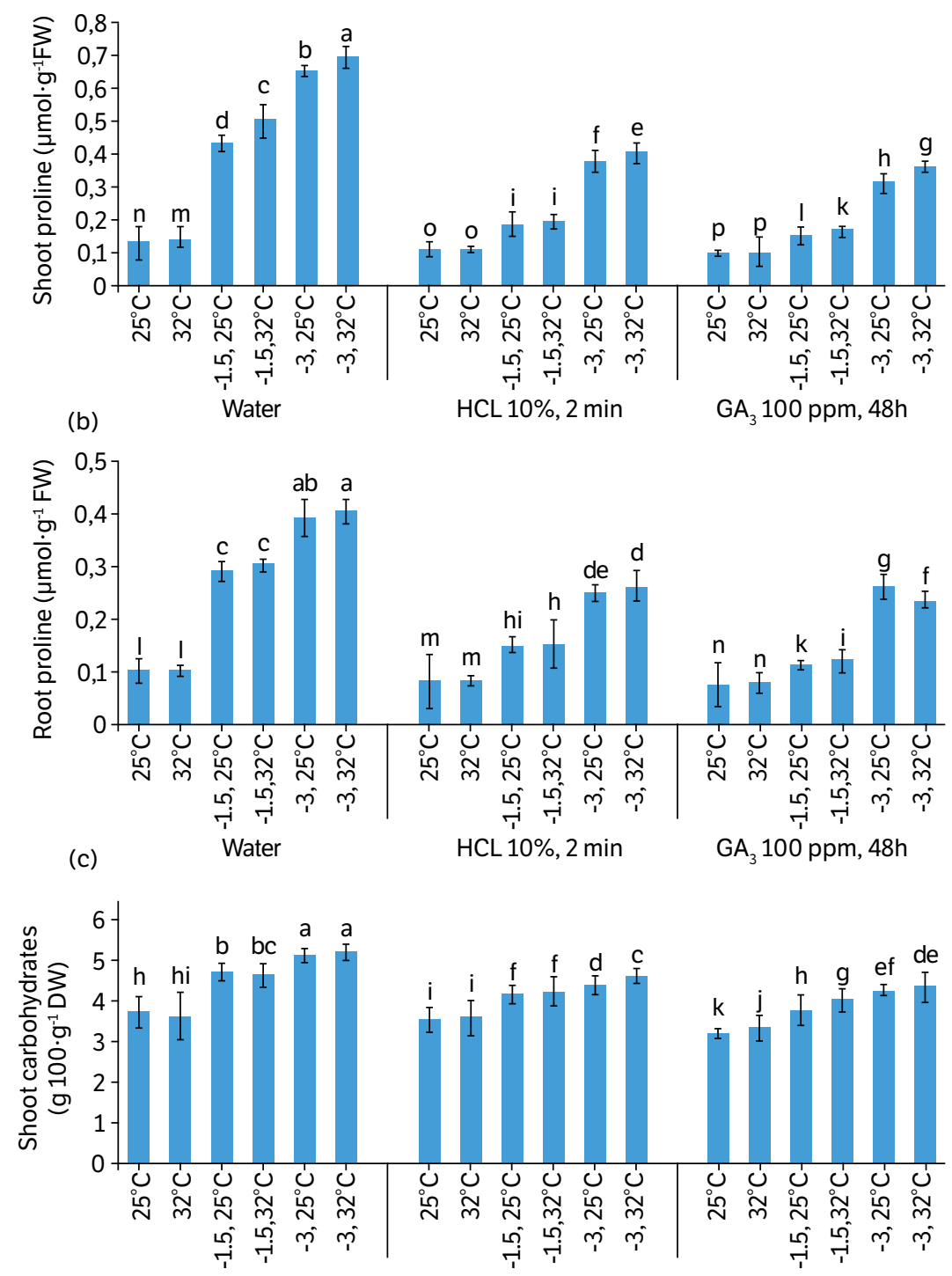

(d)

$\mathrm{HCL} 10 \%, 2 \mathrm{~min}$

$\mathrm{GA}_{3} 100 \mathrm{ppm}, 48 \mathrm{~h}$

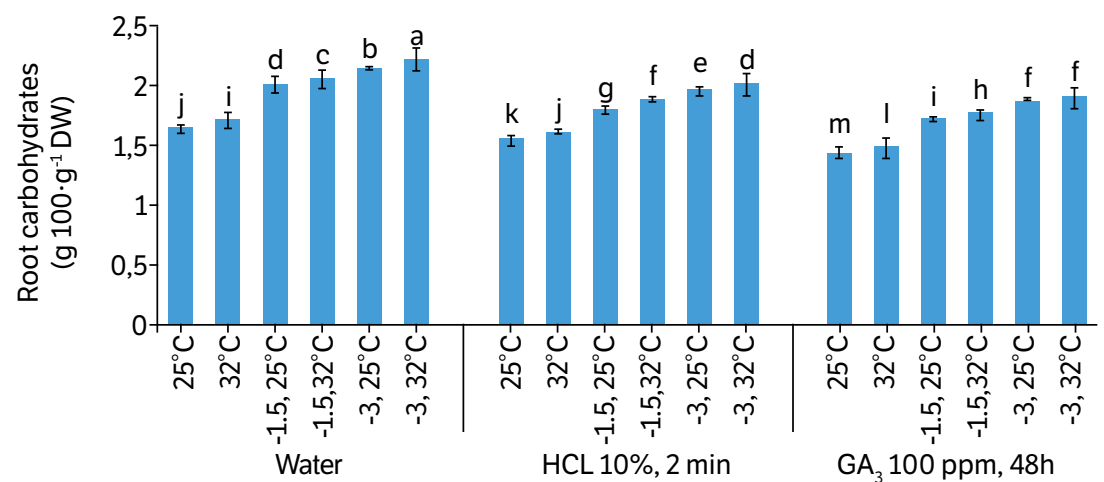

Figure 1. Influence of chemical and hormonal seeds treatments on proline and carbohydrate contents in guava under different osmotic and temperature regimes. (a) shoot free proline, (b) root free proline, (c) shoot carbohydrates, (d) root carbohydrates. Data are means \pm S.E., different letters above the bars indicate significant differences at $\mathrm{p} \leq 0.05$ (Duncan's multiple range test). $\mathrm{HCl}$ : seed soaking in $10 \% \mathrm{HCl}$ solution for $2 \mathrm{~min} ; \mathrm{GA}_{3}$ : seed priming in $0.1 \%$ gibberellic acid solution for $48 \mathrm{~h}$. 
(a)

Variables - PCA

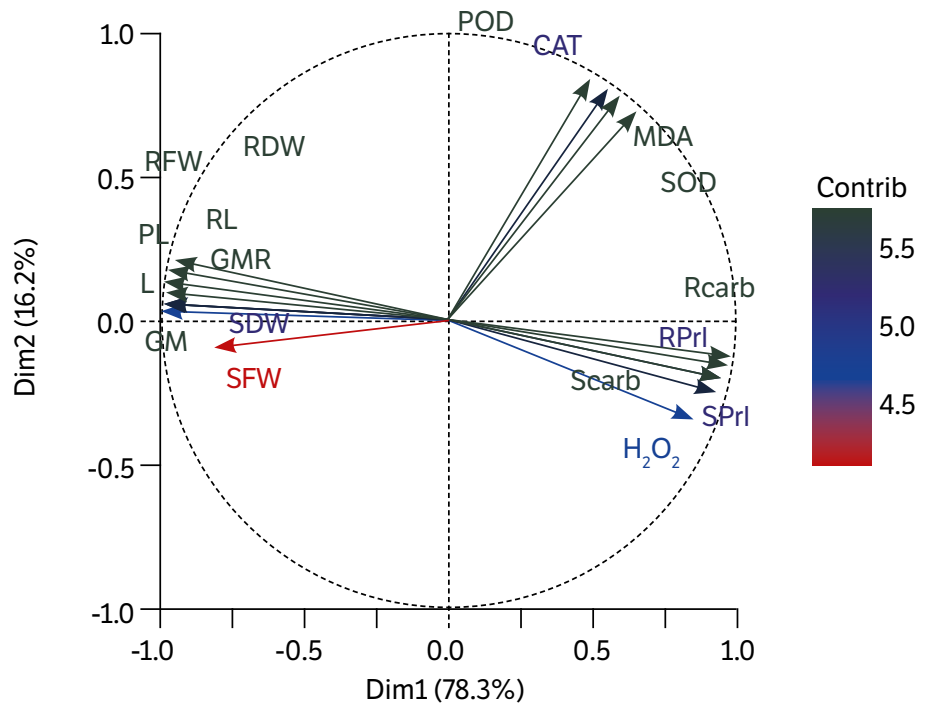

(b)

Individuals - PCA

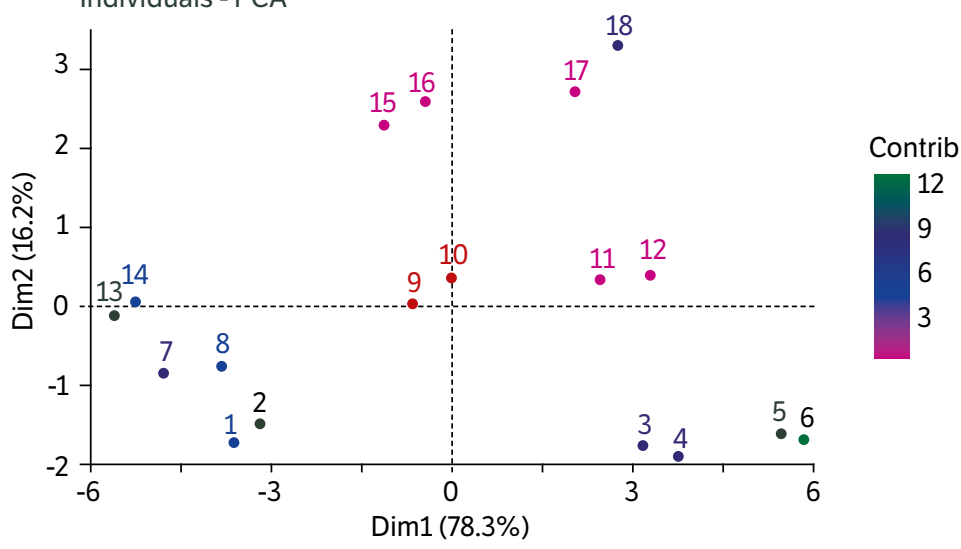

(c)

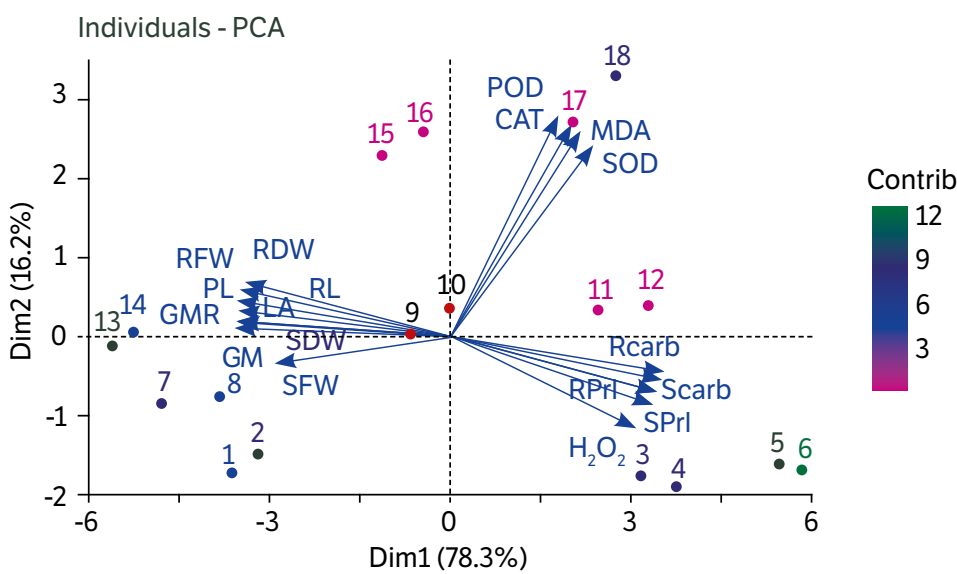

Figure 2. Principal component analysis (PCA) of guava seeds exposed to heat and osmotic stress treatments. (a) PCA loading plot for PC1 and PC2, (b) PCA individual plot for the different conditions of guava seeds, (c) PCA biplot of the treatment-variable association where the lines originating from the center indicate positive or negative correlations of different variables. 1 : Water, $\psi \mathrm{s}(0), \operatorname{Temp} .\left(25^{\circ} \mathrm{C}\right) ; 2: \mathrm{Water}, \psi \mathrm{s}(0)$, Temp. $\left(32^{\circ} \mathrm{C}\right) ; 3$ : Water, $\psi s$ ( $(-1.5)$, Temp. $\left(25^{\circ} \mathrm{C}\right)$; 4 : Water, $\psi s$ s $(-1.5)$, Temp. $\left(32^{\circ} \mathrm{C}\right)$; 5 : Water, $\psi \mathrm{s}(-3)$, Temp. $\left(25^{\circ} \mathrm{C}\right) ; 6:$ Water, $\psi \mathrm{s}(-3)$, Temp. $(32$ $\left.{ }^{\circ} \mathrm{C}\right) ; 7: \mathrm{HCl}(10 \%, 2 \mathrm{~min}), \psi s(0)$, Temp. $\left(25^{\circ} \mathrm{C}\right) ; 8: \mathrm{HCl}(10 \%, 2 \mathrm{~min}), \psi \mathrm{s}(0)$, Temp. $\left(32^{\circ} \mathrm{C}\right) ; 9: \mathrm{HCl}(10 \%, 2 \mathrm{~min}), \psi \mathrm{s}(-1.5), \mathrm{Temp} .\left(25^{\circ} \mathrm{C}\right) ; 10: \mathrm{HCl}$ (10\%, $2 \mathrm{~min}), \psi \mathrm{s}(-1.5)$, Temp. $\left(32^{\circ} \mathrm{C}\right) ; 11: \mathrm{HCl}(10 \%, 2 \mathrm{~min}), 2 \mathrm{~min}, \psi \mathrm{s}(-3)$, Temp. $\left(25^{\circ} \mathrm{C}\right) ; 12: \mathrm{HCl}(10 \%, 2 \mathrm{~min}), \psi \mathrm{s}(-3), \mathrm{Temp}^{\circ}\left(32^{\circ} \mathrm{C}\right) ; 13: \mathrm{GA}{ }_{3}$

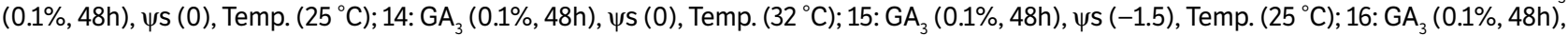

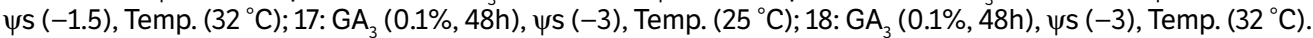


accumulation of some osmolytes and increased activity of some enzymes. The accumulation of organic osmolytes in the cytoplasm to maintain the water potential of the plant during periods of stress is one of the osmotic defense mechanisms (Farooq et al. 2009). The exposure to stress may increase the accumulation of proline, soluble sugars and other organic osmolytes in the plant shoots and roots (Farooq et al. 2018; Zahedi et al. 2019). Accumulation of these solutes led to a decrease in the water potential of olive organs and water uptake in the plant became possible (Gholami and Zahedi 2019). As an osmotic adjustment option, proline accumulates more in plants at a higher temperature and under drought stress. However, $\mathrm{GA}_{3}$ treatment may reduce the proline accumulation in plant tissues. This could stimulate vegetative growth of plant grown under drought stress (Kaya et al. 2006). Shoots (especially leaves) are a major sink for the accumulation of proline under stressful conditions (Chun et al. 2018).

Carbohydrate accumulation is induced by respiratory changes under stress conditions (Gholami and Zahedi 2019). Zawaski and Busov (2014) reported that DELLA proteins and $\mathrm{GA}_{20 x \mathrm{~s}}$ not only repress growth but enhance plant resistance to stress via activation of ROS-detoxification enzymes, increased carotenoid production as a nonenzymatic antioxidant and ABA biosynthesis. Plants alter the activity of antioxidant enzymes such as SOD, CAT, APX and POX; antioxidant enzymes and endogenous GAs to avoid drought-induced oxidative damages (Liu et al. 2013). Carbohydrate accumulation in plant tissues under stress conditions may help in effective osmoregulation. In this regard, soluble organic compounds may act as osmoprotectants in addition to their role in osmoregulation (Gill et al. 2003; Farooq et al. 2018).

\section{CONCLUSION}

Chemical and hormonal seed treatments improved seed germination and seedling growth of guava under heat and osmotic stresses by modulation of antioxidant enzymes and leaf proline. Seed treatments with $\mathrm{GA}_{3}(0.1 \%, 48 \mathrm{~h})$ were the most effective in this regard. Germination and early growth are the key to successful plant establishment under optimal and less than optimal conditions. In this study, seed treatment with $\mathrm{GA}_{3}$ was effective in improving guava seed germination and seedling growth under heat and osmotic stresses. However, further studies are needed to understand the role of hormonal homeostasis in germination under less than optimum conditions.

\section{ACKNOWLEDGMENTS}

Authors are grateful to the Agricultural and Natural Resources Research and Education Center, Bandar Abbas, Iran.

\section{AUTHOR'S CONTRIBUTION}

Conceptualization: Hosseini M. S., Fahadi Hoveizeh N. and Zahedi S. M; Methodology: Hosseini M. S. and Zahedi S. M.; Investigation: Hosseini M. S., Zahedi S. M. and Fahadi Hoveizeh N.; Writing - Original Draft: Hosseini M. S., Fahadi Hoveizeh N. and Rafiee M.; Writing - Review and Editing: Zahedi S. M., Li L. and Farooq M.

\section{REFERENCES}

Aebi, H. (1974). Catalase. In H. U. Bergmeyer (Ed.), Methods of enzymatic analysis (p. 673-677). New York: Academic Press. https://doi. org/10.1016/B978-0-12-091302-2.50032-3

Alexieva, V., Sergiev, I., Mapelli, S. and Karanov, E. (2001). The effect of drought and ultraviolet radiation on growth and stress markers in pea and wheat. Plant, Cell \& Environment, 24, 1337-1344. https://doi.org/10.1046/j.1365-3040.2001.00778.x

Arteca, R. N. (1996). Plant growth substances: Principles and applications. Boston: Springer. https://doi.org/10.1007/978-1-4757-2451-6 
Beauchamp, C. and Fridovich, I. (1971). Superoxide dismutase: Improved assays and an assay applicable to acrylamide gels. Analytical Biochemistry, 44, 276-287. https://doi.org/10.1016/0003-2697(71)90370-8

Brijwal, M. and Kumar, R. (2013). Studies on the seed germination and subsequent seedling growth of guava (Psidium guajava L.). Indian Journal of Agricultural Research, 47, 347-352.

Cavallaro, V., Maucieri, C. and Barbera, A. C., (2014). Lolium multiflorum Lam. cvs germination under simulated olive mill wastewater salinity and pH stress. Ecological Engineering, 71, 113-117. https://doi.org/10.1016/j.ecoleng.2014.07.055

Chun, S. C., Paramasivan, M. and Chandrasekaran, M. (2018). Proline accumulation influenced by osmotic stress in arbuscular mycorrhizal symbiotic plants. Frontiers in Microbiology, 9, 2525. https://doi.org/10.3389/fmicb.2018.02525

Dai, A. (2011). Drought under global warming: a review. WIREs Climate Change, 2, 45-65. https://doi.org/10.1002/wcc.81

Dawood, M. G. (2018). Stimulating plant tolerance against abiotic stress through seed priming. In A. Rakshit and H. Singh (Eds.), Advances in Seed Priming (p. 147-183). Singapore: Springer. https://doi.org/10.1007/978-981-13-0032-5_10

Dhara, P., Patel, N. L., Tanveer, A., Apeksha, P. and Kumar, V. (2017). Effect of pre-cooling packaging material on chemical and sensory quality of guava fruits [Psidium guajava (Linn.)] cv Allahabad Safeda. Environment \& Ecology, 35, 64-69.

Dhindsa, R. S., Plumb-Dhindsa, P. and Thorpe, T. A. (1981). Leaf senescence: Correlated with increased levels of membrane permeability and lipid peroxidationand decreased levels of superoxide dismutase and catalase. Journal of Experimental Botany, 32,93-101. https://doi.org/10.1093/jxb/32.1.93

Dissanayake, P., George, D. L. and Gupta, M. L. (2010). Effect of light, gibberellic acid and abscisic acid on germination of guayule (Parthenium argentatum Gray) seed. Industrial Crops and Products, 32, 111-117. https://doi.org/10.1016/j.indcrop.2010.03.012

Doijode, S. D. (2001). Guava: Psidium guajava L. In Seed storage of horticultural crops (p. 65-67). New York: Haworth.

Essien, E. P. (2004). Breaking of seed coat dormancy in guava. Tropical Science, 44, 4-42. https://doi.org/10.1002/ts.130

Fallahi, H.-R., Mohammadi, M., Aghhavani-Shajari, M. and Ranjbar, F. (2015). Determination of germination cardinal temperatures in two basil (Ocimum basilicum L.) cultivars using non-linear regression models. Journal of Applied Research on Medicinal and Aromatic Plants, 2, 140-145. https://doi.org/10.1016/j.jarmap.2015.09.004

Farooq, M., Basra, S. M. A., Khalid, M., Tabassum, R. and Mehmood, T. (2006). Nutrient homeostasis, metabolism of reserves and seedling vigor as affected by seed priming in coarse rice. Canadian Journal of Botany, 84, 1196-1202. https://doi.org/10.1139/b06-088

Farooq, M., Wahid, A., Kobayashi, N., Fujita, D. and Basra, S. M. A. (2009). Plant drought stress: effects, mechanisms and management. Agronomy for Sustainable Development, 29, 185-212. https://doi.org/10.1051/agro:2008021

Farooq, M., Ullah, A., Lee, D.-J., Alghamdi, S. S. and Siddique, K. H. M. (2018). Desi chickpea genotypes tolerate drought stress better than kabuli types by modulating germination metabolism, trehalose accumulation and carbon assimilation. Plant Physiology and Biochemistry, 126, 47-54. https://doi.org/10.1016/j.plaphy.2018.02.020

Gholami, R. and Zahedi, S. M. (2019). Identifying superior drought-tolerant olive genotypes and their biochemical and some physiological responses to various irrigation levels. Journal of Plant Nutrition, 42, 2057-2069. https://doi.org/10.1080/01904167.2019.1648672

Gill, P. K., Sharma, A. D., Singh, P. and Bhullar, S. S. (2003). Changes in germination, growth and soluble sugar contents of Sorghum bicolor (L.) Moench seeds under various abiotic stresses. Plant Growth Regulation, 40,157-162. https://doi.org/10.1023/A:1024252222376

Grzesik, M., Górnik, K., Janas, R., Lewandowki, M., Romanowska-Duda, Z. and van Duijn, B. (2017). High efficiency stratification of apple cultivar Ligol seed dormancy by phytohormones, heat shock and pulsed radio frequency. Journal of Plant Physiology, 219, 81-90. https:// doi.org/10.1016/j.jplph.2017.09.007

He, M.-X., Du, X.-F., Chen, L., Lu, X.-Y. and Lan, H.-Y. (2013). Effects of salt, alternating temperature and hormone treatments on seed germination and seedling establishment of Suaeda aralocaspica (Chenopodiaceae) dimorphic seeds. Chinese Journal of Ecology, 32, 45-51. 
Hemeda, H. M. and Klein, B. P. (1990). Effects of naturally occurring antioxidants on peroxidase activity of vegetable extracts. Journal of Food Science, 55, 184-185. https://doi.org/10.1111/j.1365-2621.1990.tb06048.x

Humphries, T., Chauhan, B. S. and Florentine, S. K. (2018). Environmental factors effecting the germination and seedling emergence of two populations of an aggressive agricultural weed; Nassella trichotoma. PLoS ONE, 13, e0199491. https://doi.org/10.1371/journal.pone.0199491

Ibrahim, M. Z. and Nermin, A. E.-S. (2001). Response of two differentially drought tolerant varieties of maize to drought stress. Pakistan Journal of Biological Sciences, 4, 779-784. https://doi.org/10.3923/pjbs.2001.779.784

Jaleel, C. A., Manivannan, P., Wahid, A., Farooq, M., Al-Juburi, H. J., Somasundaram, R. and Pannerselvam, R. (2009). Drought stress in plants: a review on morphological characteristics and pigments composition. International Journal of Agriculture and Biology, 11, $100-105$.

Kalyani, M., Bharad, S. G. and Parameshwar, P. (2014). Effect of growth regulators on seed germination in guava. International Journal on Biological Sciences, 5, 81-91.

Kaya, C., Tuna, A. L. and Alfredo, A. C. A. (2006). Gibberellic acid improves water deficit tolerance in maize plants. Acta Physiologiae Plantarum, 28, 331-337. https://doi.org/10.1007/s11738-006-0029-7

Khare, T., Desai, D. and Kumar, V. (2012). Effect of $\mathrm{MgCl}_{2}$ stress on germination, plant growth, chlorophyll content, proline content and lipid peroxidation in sorghum cultivars. Journal of Stress Physiology \& Biochemistry, 8, 169-178.

Khosravi, M., Sahebzadeh, N., Kolyaie, R. and Mokhtari, A. (2018). Field evalution of controling methods of mango fruit flies Bactrocera zonata (Diptera: Tephritidae) in the southern part of Iran. Trakia Journal of Science, 1, 62-69. https://doi.org/10.15547/tjs.2018.01.010

Kim, D. H. (2019). Practical methods for rapid seed germination from seed coat-imposed dormancy of Prunus yedoensis. Scientia Horticulturae, 243, 451-456. https://doi.org/10.1016/j.scienta.2018.08.039

Lamichhane, J. R., Debaeke, P., Steinberg, C., You, M. P., Barbetti, M. J. and Aubertot, J.-N. (2018). Abiotic and biotic factors affecting crop seed germination and seedling emergence: a conceptual framework. Plant and Soil, 432, 1-28. https://doi.org/10.1007/s11104-018-3780-9

Lester, D. C., Carter, O. G., Kelleher, F. M. and Laing, D. R. (2002). The effect of gibberellic acid on apparent photosynthesis and dark respiration of simulated swards of Pennisetum clandestinum Hochst. Australian Journal of Agricultural Research, 23, 205-213. https://doi.org/10.1071/AR9720205

Li, Z., Xu, J., Gao, Y., Wang, C., Guo, G., Luo, Y., Huang, Y., Hu, W., Sheteiwy, M. S., Guan, Y. and Hu, J. (2017). The synergistic priming effect of exogenous salicylic acid and $\mathrm{H}_{2} \mathrm{O}_{2}$ on chilling tolerance enhancement during maize (Zea mays L.) seed germination. Frontiers in Plant Science, 8, 1153. https://doi.org/10.3389/fpls.2017.01153

Liu, T., Zhu, S., Fu, L., Yu, Y., Tang, Q. and Tang, S. (2013). Morphological and physiological changes of ramie (Boehmeria nivea L. Gaud) in

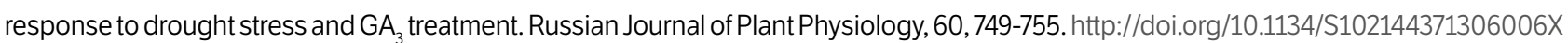

Ma, Y., Zhnng, J., Li, X., Zhang, S. and Lan, H. (2016). Effects of environmental stress on seed germination and seedling growth of Salsola ferganica (Chenopodiaceae). Acta Ecologica Sinica, 36, 456-463. https://doi.org/10.1016/j.chnaes.2016.09.008

Maldonado-Arciniegas, F., Ruales, C., Caviedes, M., Ramírez, D. X. and León-Reyes, A. (2018). An evaluation of physical and mechanical scarification methods on seed germination of Vachellia macracantha (Humb. \& Bonpl. ex Willd.) Seigler \& Ebinger. Acta Agronómica, 67, 120-125. https://doi.org/10.15446/acag.v67n1.60696

Michel, B. E. and Kaufmann, M. R. (1973). The osmotic potential of polyethylene glycol 6000. Plant Physiology, 51, 914-916. https://doi. org/10.1104/pp.51.5.914

Muhie, S. H. (2018). Seed priming with phytohormones to improve germination under dormant and abiotic stress conditions. Advances in Crop Science and Technology, 6, 403. https://doi.org/10.4172/2329-8863.1000403

Paleg, L., Aspinall, D., Coombe, B. and Nichols, P. (1964). Physiological effects of gibberellic acid. VI. Other gibberellins in three test systems. Plant Physiology, 39, 286-290. https://doi.org/10.1104/pp.39.2.286 
Ranal, M. A., Santana, D. G., Ferreira, W. R. and Mendes-Rodrigues, C. (2009). Calculating germination measurements and organizing spreadsheets. Brazilian Journal of Botany, 32, 849-855. https://doi.org/10.1590/S0100-84042009000400022

Rao, M. J., Hussain, S., Anjum, M. A., Saqib, M., Ahmad, R., Khalid, M. F., Sohail, M., Nafees, M., Ali, M. A., Ahmad, N., Zakir, I. and Ahmad, S. (2019). Effect of seed priming on seed dormancy and vigor. In M. Hasanuzzaman, V. Fotopoulos (Eds.), Priming and pretreatment of seeds and seedlings (p. 136-142). Singapore: Springer. https://doi.org/10.1007/978-981-13-8625-1_6

Santos, M. A. C., Queiroz, M. A., Bispo, J. S. and Dantas, B. F. (2015). Seed germination of Brazilian guava (Psidium guineense Swartz.). Journal of Seed Science, 37, 214-221. https://doi.org/10.1590/2317-1545v37n4152933

Salehi Sardoei, A., Shahadadi, F., Shahdadneghad, M. and Imani Fallah, A. (2014). The effect of gibberellic acid on reducing sugar of Jerusalem cherry (Solanum pseudocapsicum L.) plant. International Journal of Advanced Biological and Biomedical Research, 3, $690-695$.

Shaukat, F., Balal, R. M., Shahid, M. A., Akhtar, G., Akram, A., Khan, M. W. and Zubair, M. (2015). Identification of drought induced drastic effects on Guava (Psidium guajava) at seedling stage. International Journal of Chemical and Biochemical Sciences, 8, 15-27.

Shekafandeh, A. and Khoushkhoui, M. (2005). Micropropagation of Iranian cultivars of white flesh and red flesh of guava (Psidium guajava L.). Iranian Journal of Horticultural Science and Technology, 6, 15-26.

Sheteiwy, M. S., Gong, D., Gao, Y., Pan, R., Hu, J. and Guan, Y. (2018). Priming with methyl jasmonate alleviates polyethylene glycolinduced osmotic stress in rice seeds by regulating the seed metabolic profile. Environmental and Experimental Botany, 153, $236-248$. https://doi.org/10.1016/j.envexpbot.2018.06.001

Slama, I., Abdelly, C., Bouchereau, A., Flowers, T. and Savouré, A. (2015). Diversity, distribution and roles of osmoprotective compounds accumulated in halophytes under abiotic stress. Annals of Botany, 115, 433-447. https://doi.org/10.1093/aob/mcu239

Sourabh, J. R. S., Baloda, S. and Beniwal, V. (2018). Seedling growth pattern of guava (Psidium guajava L.) as influenced by different seed scarification treatments. International Journal of Economic Plants, 5, 131-136. https://doi.org/10.23910/IJEP/2018.5.3.023

Tanaka-Oda, A., Kenzo, T. and Fukuda, K. (2009). Optimal germination condition by sulfuric acid pretreatment to improve seed germination of Sabina vulgaris Ant. Journal of Forest Research, 14, 251-256. https://doi.org/10.1007/s10310-009-0129-5

Yemm, E. W. and Willis, A. J. (1954). The estimation of carbohydrates in plant extracts by anthrone. Biochemical Journal, 57, 508-514. https://doi.org/10.1042/bj0570508

Zahedi, S. M., Abdelrahman, M., Hosseini, M. S., Fahadi Hoveizeh, N. and Tran, L.-S. P. (2019). Alleviation of the effect of salinity on growth and yield of strawberry by foliar spray of selenium-nanoparticles. Environmental Pollution, 253, 246-258. https://doi.org/10.1016/j. envpol.2019.04.078

Zawaski, C. and Busov, V.B. (2014). Roles of gibberellin catabolism and signaling in growth and physiological response to drought and short-day photoperiods in Populus trees. PLoS ONE, 9, e86217. https://doi.org/10.1371/journal.pone.0086217

Zhang, F., Yu, J., Johnston, C. R., Wang, Y., Zhu, K., Lu, F. and Zhang, Z., Zou, J. (2015). Seed priming with polyethylene glycol induces physiological changes in sorghum (Sorghum bicolor L. Moench) seedlings under suboptimal soil moisture environments. PLoS ONE, 10, e0140620. https://doi.org/10.1371/journal.pone.0140620

In the article Improving seed germination and seedling growth of guava under heat and osmotic stresses by chemical and hormonal seed treatments with DOI: https://doi.org/10.1590/1678-4499.20200155, published in Bragantia vol.79 no.4 Campinas Oct./Dec. 2020:

In the footline where is read Bragantia, Campinas, v. 79, n. 4, p.387-399, 2020 should be read Bragantia, Campinas, v. 79, n. 4, p.512-524, 2020. 